\title{
Pressure Dependence of the Thermodynamic Critical Field in Francium
}

\author{
P.W. PACh, R. SzCZęŚNIAK AND A.P. DURAJSKI* \\ Institute of Physics, Częstochowa University of Technology, al. Armii Krajowej 19, 42-200 Częstochowa, Poland
}

\begin{abstract}
In the paper, the values of the thermodynamic critical field $\left(H_{\mathrm{C}}\right)$ for francium have been calculated. It has been assumed the wide range of the pressure: $p \in\langle 7.2 ; 14\rangle \mathrm{GPa}$. The analysis has been performed in the framework of the strong-coupling formalism. It has been predicted that the value of the ratio $H_{\mathrm{C}}(0) / \sqrt{\rho(0)}$ increases with the increasing pressure from $3.08 \mathrm{meV}$ to $5.84 \mathrm{meV}$, where $\rho(0)$ denotes the electron density of states at the Fermi level. The dimensionless parameter $T_{\mathrm{C}} C^{\mathrm{N}}\left(T_{\mathrm{C}}\right) / H_{\mathrm{C}}^{2}(0)$ is smaller than in the BCS theory and decreases with pressure from 0.150 to 0.141 . The symbol $T_{\mathrm{C}}$ represents the critical temperature and $C^{\mathrm{N}}$ is the specific heat for the normal state.
\end{abstract}

DOI: 10.12693/APhysPolA.127.231

PACS: 74.20.Fg, 74.25.Bt, 74.62.Fj

\section{Introduction}

Francium is the most rarest and unstable of the naturally occurring elements. The longest lived francium isotopes are ${ }^{212} \mathrm{Fr}$ and ${ }^{223} \mathrm{Fr}$ which have half-lives of about 20 min [1]. It is a highly radioactive alkali metal similar to cesium. Compared to other elements, knowledge of the physical and chemical properties of francium is not large enough.

The recent theoretical studies predict existence of superconductivity in compressed Fr with the critical temperature $\left(T_{\mathrm{C}}\right)$ reaching a value of about $7 \mathrm{~K}$ at $14 \mathrm{GPa}$ [2]. The performed calculations of the electronic structure using density functional theory show pronounced effect of pressure on physical properties of Fr. Under small pressure we can see a phase transition from hcp to bcc, which is stable up to $14 \mathrm{GPa}$. Moreover, the electronphonon coupling constant $(\lambda)$ and critical temperature significantly increase with pressure.

In this paper, we theoretically studied the magnetic properties of francium under high pressure: $p \in$ $\langle 7.2 ; 14\rangle$ GPa. In particular, using the strong-coupling Eliashberg theory of superconductivity, we investigated the pressure and temperature effect on the thermodynamic critical field.

\section{Calculations and results}

The central quantity of the Eliashberg theory is the Eliashberg spectral function $\left(\alpha^{2} F(\Omega)\right)$, which expresses the electron-phonon interaction in the form of a spectral density. The $\alpha^{2} F(\Omega)$ can be obtained from the ab initio calculations or it can also be derived, from experiments by inversion of tunnelling spectra and then used as input

\footnotetext{
${ }^{*}$ corresponding author; e-mail: adurajski@wip.pcz.pl
}

in the Eliashberg equations to determine the thermodynamic properties of superconducting state. The Eliashberg equations on the imaginary-axis for the order parameter $\left(\Delta_{n}=\phi_{n} / Z_{n}\right)$ and the wave function renormalization factor $\left(Z_{n}\right)$ take the following form [3-5]:

$$
\begin{aligned}
& \phi_{n}= \\
& \frac{\pi}{\beta} \sum_{m=-M}^{M} \frac{K\left(\mathrm{i} \omega_{n}-\mathrm{i} \omega_{m}\right)-\mu^{\star} \theta\left(\omega_{\mathrm{C}}-\left|\omega_{m}\right|\right)}{\sqrt{\omega_{m}^{2} Z_{m}^{2}+\phi_{m}^{2}}} \phi_{m}, \\
& Z_{n}=1+\frac{1}{\omega_{n}} \frac{\pi}{\beta} \sum_{m=-M}^{M} \frac{K\left(\mathrm{i} \omega_{n}-\mathrm{i} \omega_{m}\right)}{\sqrt{\omega_{m}^{2} Z_{m}^{2}+\phi_{m}^{2}}} \omega_{m} Z_{m},
\end{aligned}
$$

where $\omega_{\mathrm{C}}$ denotes the cut-off frequency $\left(\omega_{\mathrm{C}}=10 \Omega_{\max }\right)$, $\theta$ is the Heaviside function, $n$-th Matsubara frequency is derived from the expression: $\omega_{n} \equiv \pi k_{\mathrm{B}} T(2 n-1)$, with $n=0, \pm 1, \pm 2, \ldots$ and $k_{\mathrm{B}} T$ being the temperature in energy units $\left(k_{\mathrm{B}}\right.$ represents the Boltzmann constant). The Coulomb pseudopotential $\left(\mu^{\star}\right)$ describes the effects of the electron repulsion. It is a parameter that must be fitted in order to reproduce the experimental data. Due to the absence of experimental results for superconducting francium, we take the commonly accepted values of Coulomb pseudopotential: $\mu^{\star}=0.1$.

The electron-phonon pairing kernel is defined as:

$$
K\left(\mathrm{i} \omega_{n}-\mathrm{i} \omega_{m}\right) \equiv \lambda \frac{\Omega_{\mathrm{C}}^{2}}{\left(\omega_{n}-\omega_{m}\right)^{2}+\Omega_{\mathrm{C}}^{2}},
$$

where the symbol $\Omega_{\mathrm{C}}$ represents the characteristic phonon frequency, whose value has been chosen so that the critical temperature estimated on the basis of the Eliashberg equations would be in a good agreement with the value presented in the paper [2]. The Eliashberg equations have been solved numerically for 2201 Matsubara frequencies $(M=1100)$ using a numerical method presented in the papers $[6,7]$ and successfully used in the recent studies [8-10].

In Eq. (3), the electron-phonon coupling constant can be expressed: 


$$
\lambda \equiv 2 \int_{0}^{\infty} \mathrm{d} \Omega \frac{\alpha^{2} F(\Omega)}{\Omega} .
$$

It is worth emphasizing that the Eliashberg theory can be successfully applied if the electron-phonon coupling is strong $(\lambda \geq 0.5)$. In the case of francium the value of $\lambda$ increases from 0.94 to 1.13 for investigating range of pressure [2].

Solutions of Eqs. (1) and (2) can be used directly to compute the free energy difference between the superconducting and normal state [11]:

$$
\begin{aligned}
& \frac{\Delta F}{\rho(0)}=-2 \pi k_{\mathrm{B}} T \sum_{n=1}^{M}\left(\sqrt{\omega_{n}^{2}+\Delta_{n}^{2}}-\left|\omega_{n}\right|\right) \\
& \quad \times\left(Z_{n}^{S}-Z_{n}^{\mathrm{N}} \frac{\left|\omega_{n}\right|}{\sqrt{\omega_{n}^{2}+\Delta_{n}^{2}}}\right),
\end{aligned}
$$

where $\rho(0)$ denotes the electron density of states at the Fermi level. The functions $Z_{n}^{S}$ and $Z_{n}^{\mathrm{N}}$ represent the wave function renormalization factors for the superconducting and normal state, respectively.

Then, the thermodynamic critical field can be calculated with the help of the following expression [12] (cgs units):

$$
\frac{H_{\mathrm{C}}}{\sqrt{\rho(0)}}=\sqrt{-8 \pi[\Delta F / \rho(0)]} .
$$

The results obtained for compressed francium are presented in Fig. 1.

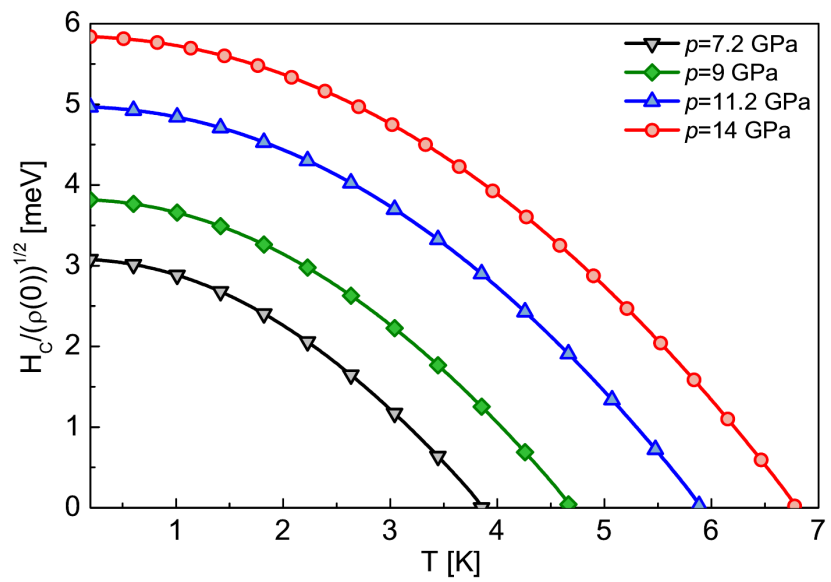

Fig. 1. The thermodynamic critical field as a function of the temperature for selected values of pressure.

We can note that with the temperature increases the value of $H_{\mathrm{C}}(T) / \sqrt{\rho(0)}$ noticeably decreases and reaches zero at $T_{\mathrm{C}}$. On the other hand, the applied pressure causes an increase of $H_{\mathrm{C}}(0) / \sqrt{\rho(0)}$ from $3.08 \mathrm{meV}$ to $5.84 \mathrm{meV}$, where $H_{\mathrm{C}}(0)=H_{\mathrm{C}}\left(T_{0}\right)$ and $T_{0}=0.2 \mathrm{~K}$. It should be noted that this result is related to the growth of the electron-phonon coupling constants with an increase of pressure (see Fig. 2).

In the last step, the values of the dimensionless ratio $R_{H} \equiv T_{\mathrm{C}} C^{\mathrm{N}}\left(T_{\mathrm{C}}\right) / H_{\mathrm{C}}^{2}(0)$ has been calculated. Symbol $C^{\mathrm{N}}$ denotes the specific heat in the normal state:

$$
\begin{aligned}
C^{\mathrm{N}} & =\gamma T, \text { where } \gamma \text { is the Sommerfeld constant }[13,14] \\
\gamma & \equiv \frac{2 \pi^{2} k_{\mathrm{B}}^{2}}{3} \rho(0)(1+\lambda)
\end{aligned}
$$

The Eliashberg approach, in contrast to the BCS theory [15], takes into account a strong electron-phonon coupling and retardation effects, so it would be expected to reach for Fr a value of $R_{H}$ lower than 0.168 (BCS prediction). Indeed, this situation is clearly visible to examined element, where $R_{H} \in\langle 0.150 ; 0.141\rangle$. The exact results are presented in Fig. 2.

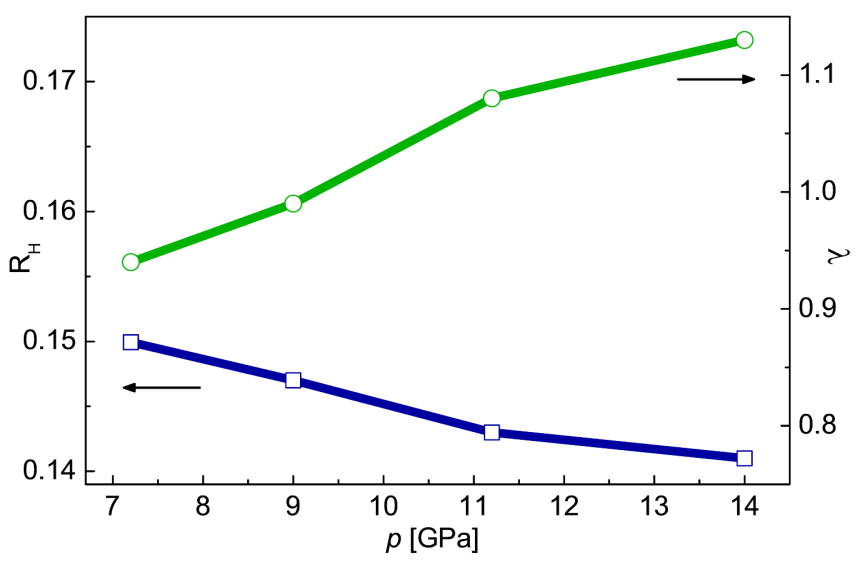

Fig. 2. The electron-phonon coupling constant (open circle) and the $R_{H}$ ratio (open square) as a function of the pressure.

The similar result was observed in the radioactive $\alpha$ phase of polonium (Po) at the reduced volume $\left(V / V_{\exp }=\right.$ $0.93)$, where $\left[R_{H}\right]_{\mathrm{Po}}=0.147[9]$. Let us note that also critical temperature and thermodynamic critical field for Po clearly correspond to the results obtained for francium at $14 \mathrm{GPa}$, in particular: $\left[T_{\mathrm{C}}\right]_{\mathrm{Po}}=7.11 \mathrm{~K}$ and $\left[H_{\mathrm{C}}(0) / \sqrt{\rho(0)}\right]_{\mathrm{Po}}=5.95 \mathrm{meV}[9]$.

\section{Conclusions}

In presented paper we discussed the effect of high pressure $(p \in\langle 7.2 ; 14\rangle)$ on the superconducting properties of francium within the framework of Eliashberg theory. In particular, the presented model enables us to study the pressure behaviors of the thermodynamic critical field and $R_{H}$ ratio. Based on carried out calculations we found that thermodynamic critical field at the temperature close to absolute zero $\left(H_{\mathrm{C}}(0) / \sqrt{\rho(0)}\right)$ increases with pressure from $3.08 \mathrm{meV}$ to $5.84 \mathrm{meV}$. On the other hand, $R_{H}$ ratio decreases from 0.150 to 0.141 .

\section{Acknowledgments}

Computational calculations were performed at the Poznan Supercomputing and Networking Center. 


\section{References}

[1] C. Fry, M. Thoennessen, At. Data Nucl. Data 99, 497 (2013).

[2] A.P. Koufos, D.A. Papaconstantopoulos, Int. J. Quantum Chem. 113, 2070 (2013).

[3] G.M. Eliashberg, Sov. Phys. JETP 11, 696 (1960).

[4] D.J. Scalapino, in: Superconductivity, Ed. R.D. Parks, Marcel Dekker, New York 1969.

[5] P.B. Allen, B. Mitrovic, in: Solid State Physics, Eds. F. Seitz, D. Turnbull, H. Ehrenreich, Academic Press, New York 1982.

[6] R. Szczęśniak, Acta Phys. Pol. A 109, 179 (2006).

[7] R. Szczęśniak, Solid State Commun. 138, 347 (2006).

[8] R. Szczęśniak, D. Szczęśniak, K.M. Huras, Phys. Status Solidi B 251, 178 (2014).
[9] R. Szczęśniak, A.P. Durajski, P.W. Pach, J. Phys. Chem. Solids 75, 224 (2014).

[10] D. Szczęśniak, R. Szczęśniak, Acta Phys. Pol. A 127, 254 (2014).

[11] J. Bardeen, M. Stephen, Phys. Rev. 136, A1485 (1964).

[12] J.P. Carbotte, Rev. Mod. Phys. 62, 1027 (1990).

[13] C. Kittel, Introduction to Solid State Physics, 8th ed., Wiley, New York 2005.

[14] C.S. Lue, H.F. Liu, C.N. Kuo, P.S. Shih, J.-Y. Lin, Y.K. Kuo, M.W. Chu, T.-L. Hung, Y.Y. Chen, Supercond. Sci. Technol. 26, 055011 (2013).

[15] J. Bardeen, L.N. Cooper, J.R. Schrieffer, Phys. Rev. 108, 1175 (1957). 